\title{
Evaluation of pericardial hydatid cysts by different echocardiographic imaging modalities
}

\author{
Attila Nemes $\cdot$ Marcel L. Geleijnse - Robert-Jan van Geuns $\cdot$ Kadir Caliskan • \\ Michelle Michels · Osama I. I. Soliman · Jackie S. McGhie · Folkert J. ten Cate
}

Received: 18 January 2006/ Accepted: 9 March 2006/Published online: 20 April 2006

(C) Springer Science+Business Media B.V. 2006

\begin{abstract}
Cardiac hydatid disease or echinococcosis is a rare complication of Echinococcus granulosus infection. Traditionally, the diagnosis is made with two-dimensional transthoracic echocardiography. This case report shows the complementary function of the different echo modalities (two-dimensional, three-dimensional, and contrast echocardiography) and MRI in the evaluation of pericardial hydatid cyst.
\end{abstract}

Keywords Echocardiography · Hydatid cyst · Pericardium

\section{Introduction}

Cardiac hydatid disease or echinococcosis is a rare complication of Echinococcus granulosus

Dr. Attila Nemes is a visiting fellow from the University of Szeged (Hungary) and is supported by the "Research Fellowship of the European Society of Cardiology".

Dr. Osama Soliman is a visiting fellow from the Al Azhar University (Cairo, Egypt) and is supported by the Egyptian Government.

A. Nemes · M. L. Geleijnse · R.-J. van Geuns ·

K. Caliskan · M. Michels · O. I. I. Soliman ·

J. S. McGhie · F. J. ten Cate $(\varangle)$

Department of Cardiology, Thoraxcenter, Erasmus

Medical Center Rotterdam, Dr Molewaterplein 40,

Room Ba304, 3015 GD Rotterdam, The Netherlands

e-mail: f.j.tencate@erasmusmc.nl

Tel.: +31-10-4635669

Fax: $+31-10-4635498$ infection. Cardiopericardial involvement is seen in approximately $2 \%$ of all patients [1]. Traditionally, the cardiac diagnosis is made with 2dimensional transthoracic echocardiography, showing the presence of a cystic mass within the myocardium or pericardium that may become calcified with multiple septae and multitextual hypoechoic contents [2]. Transesophageal studies may image the cysts in more detail [3, 4]. Newer echocardiographic imaging modalities such as contrast echocardiography and three-dimensional echocardiography may also add in the diagnosis of these cysts [2, 5]. Currently, the gold standard to assess cysts is magnetic resonance imaging (MRI) and computer tomography (CT) [6, 7].

\section{Case report}

We present a case of a 24 year-old man, whose medical history started in 1999 in Marocco with cardiac tamponade, pleural effusion, ascites and liver dysfunction. Two-dimensional echocardiography showed multiple cystic structures in the pericardium, one of which most likely had ruptured and caused the symptoms. A large amount of serosanguinolent fluid (1.6 1) was evacuated by pericardiocentesis. Laboratory results were negative for tuberculosis, but positive for Echinococcus granulosus. In 2002, while still in Marocco, severe left ventricular dysfunction developed and the patient was treated with ACE-inhibition, 
diuretics, digoxin, and intermittent dobutamine infusions. In 2003 the patient discontinued therapy and he was seen at our outpatient clinic because of progressive shortness of breath. At physical examination the patient showed evidence of increased filling pressures and treatment with ACE-inhibition and diuretics was re-initiated. Transthoracic echocardiography showed a dilated cardiomyopathy (left ventricular end-diastolic diameter $70 \mathrm{~mm}$, ejection fraction 30\%) with moderate central mitral regurgitation. In addition to this, two extra-cardiac structures bulging into the right atrium and right ventricle were seen (Fig. 1). With SonoVue ${ }^{\circledR}$ contrast transthoracic echocardiography the cystic structures were not opacified with contrast (Fig. 2). Additional imaging with real-time three-dimensional echocardiography with a Sonos 7500 system (Philips, Best, the Netherlands) and TomTec 4D Echo 5.3 workstation (TomTec Inc., Unterschleissheim, Germany) confirmed the presence of two cysts in the pericardial cavity near to the right atrial and ventricular walls $(34 \times 34 \times 31 \mathrm{~mm}$ and $36 \times 30 \times 33 \mathrm{~mm}$ ) (Fig. 3). Magnetic resonance imaging (GE Signa CVi 1.5 T, Milwaukee, USA) confirmed the correct location of the cysts and the connections to adjacent structures (Fig. 4). After unsuccessful treatment with albendazole $800 \mathrm{mg}$ bid for more 2 years, all cystic structures were surgically removed in 2005 .

\section{Discussion}

An accurate diagnosis of hydatid cyst is essential for prompt management of patients with echinococcosis. This case report shows the complementary function of different echo modalities. Two-dimensional transthoracic echocardiography identified two structures, suspected for hydatid cysts. With contrast echocardiography the definite diagnosis of an extra-cardiac structure was confirmed, non-opacified structures were identified as extra-cardiac [2]. Real-time three-dimensional echocardiography allowed a more precise evaluation of the cystic size, shape and structure, permitted anyplane analysis and helped to clarify its relations to neighbouring structures.

Echinococcosis is caused by E. granulosus (hydatid disease) and E. multilocularis (alveolar echinococcosis) [8]. The hydatid cyst is a tumor caused by development of the embryonic form of the E. granulosus. The treatment for cardiopericardial hydatid cysts begins with antiparasitic medications, but frequently requires complete surgical removal. A broad spectrum of antihelmintics containing fluoromebendazole and mebendazole has been tried, but only flubendazole ( $2 \mathrm{~g}$ daily) is recommended because the other antihelmintics suffer from toxic side effects due to the need for high doses [9]. Surgery is recommended if the cyst is large, not calcified, causing symptoms or adverse effects on hemodynamics or blood supply to the heart, and especially when the hydatid serologic tests are positive [1]. Pre-operative echocardiographic assessment of the cyst may be helpful in guiding surgery. E. multilocularis heart involvement appears exceedingly rare, only a few studies reported isolated pericardial involvement due to E. multilocularis [10]. The Em2 plus ELISA test has a high sensitivity (97\%)

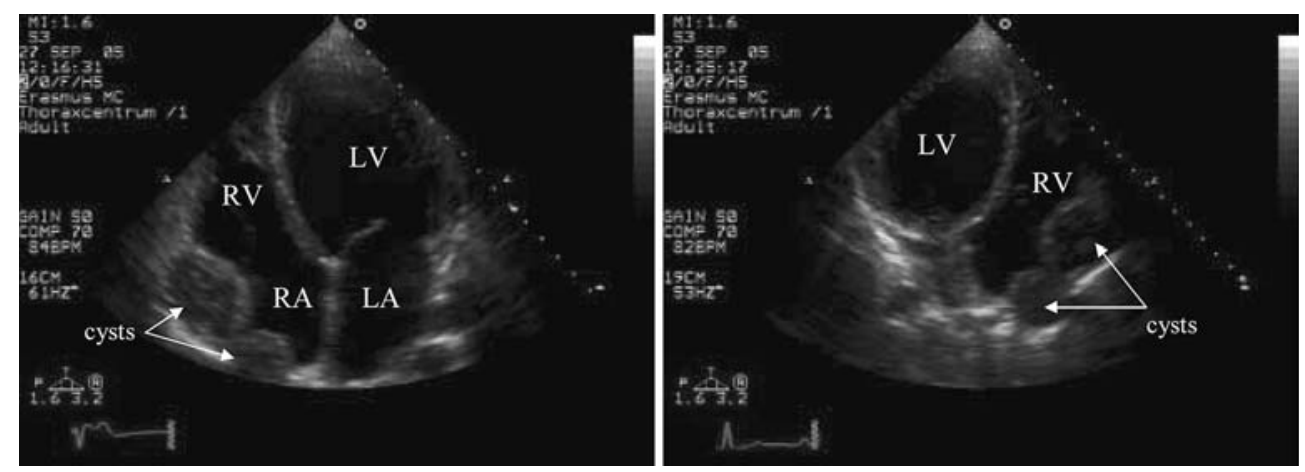

Fig. 1 Two-dimensional transthoracic echocardiography showing two extra-cardiac structures bulging into the right atrium and ventricle. Abbreviations: LA: left atrium, LV: left ventricle, RA: right atrium, RV: right ventricle 
Fig. 2 SonoVue ${ }^{\circledR}$ contrast transthoracic echocardiography showing absence of opacification of the structures. Abbreviations as in Figure 1

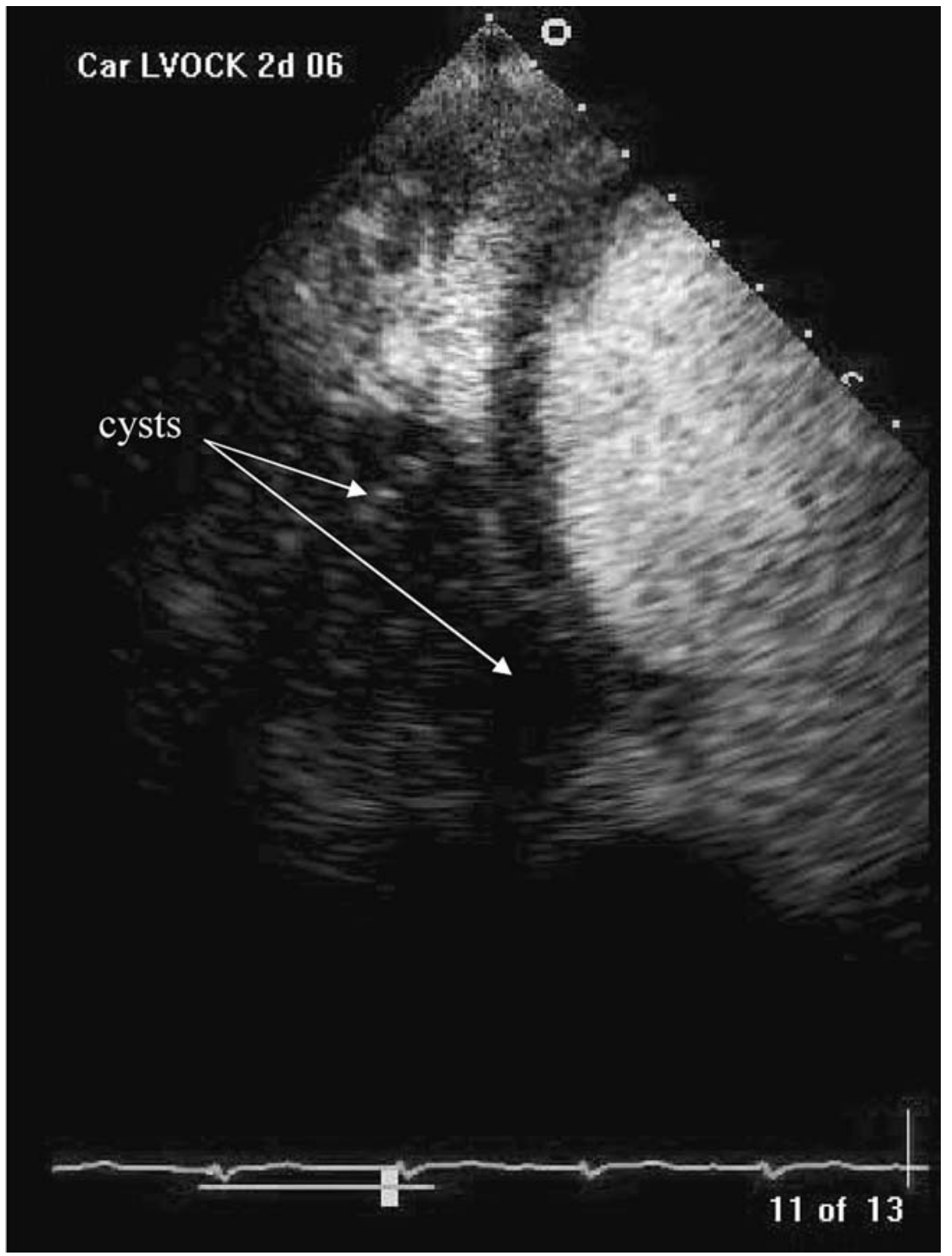

and specificity (99\%) for the diagnosis of E. multilocularis infection [11]. Sensitivity of the parasitic lesions to albendazole in this location suggests that chemotherapy should be considered when surgery is impossible or carries a high risk. Because disease improvement may occur very late, albendazole therapy should be continued even when no change is present over the first years of treatment. Despite objective mass regression and encouraging immunological changes, the decision to withdraw albendazole remains difficult. Appropriate tools are still needed to measure the viability of the parasitic lesions in order to avoid an indefinite treatment in such patients [10].

Classic echocardiographic features include the presence of a cystic mass within the myocardium or pericardium that may become calcified with multiple septae and multitextual hypoechoic contents. Echocardiography can show the relation between the cyst and the cavities, any obstruction of the valves or effects on ventricular function. Although serology tests are commonly used, echocardiography is more sensitive than serology, when isolated cardiac echinococcosis is suspected. With contrast echocardiography the definite 

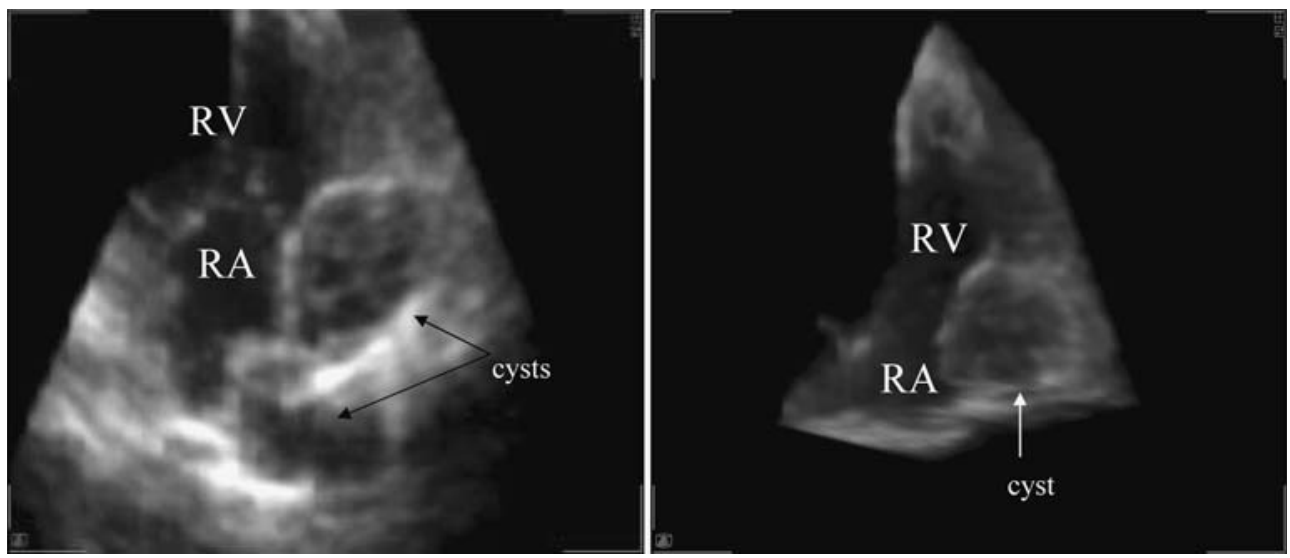

Fig. 3 Real-time 3-dimensional echocardiography allows a more precise evaluation of the cystic size, shape and structure and permits anyplane analysis. Abbreviations as in Figure 1

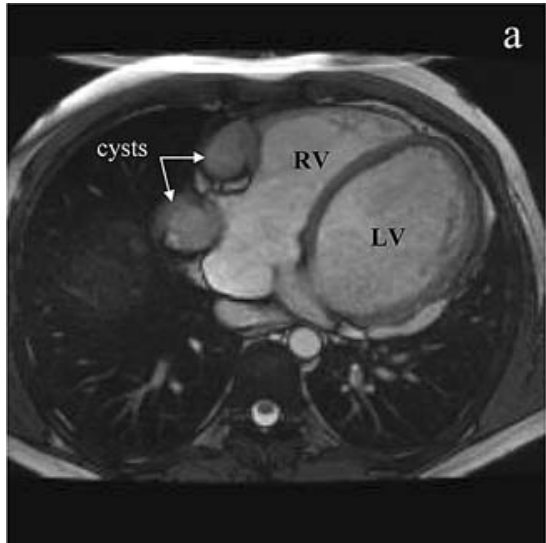

Fig. 4 Magnetic resonance imaging confirmation of the correct cyst location and connections to adjacent structures. Figure $4 \mathbf{a}$ is a diastolic still frame from a steady-state

diagnosis of an extra-cardiac structure can be confirmed. Ultrasound contrast agent microbubbles pass the pulmonary circulation and opacify all cavities connected to the heart, so non-opacified structures are identified as extra-cardiac [2]. The recently developed real-time 3D echocardiography is an easy-to-learn method helping us in the spatial evaluation of cysts and to clarify relations to adjacent structures [5].

In the real practice, indications for CT or MRI are restricted to extra-abdominal disease, patients not suited for ultrasonography (obesity or meteorism, complicated cysts and planning of surgery or interventional therapy). MRI can be used as 'gold standard' in the diagnosis of hydatid cyst,

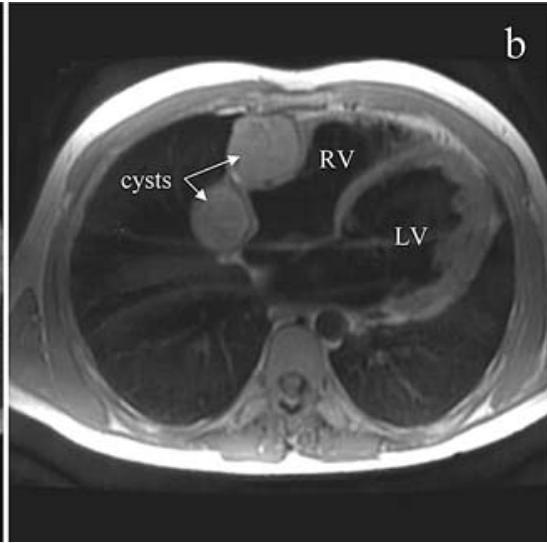

free-precession technique, Figure $4 \mathbf{b}$ is a breathhold blackblood fast spin echo technique. Abbreviations as in Figure 1

but this imaging modality is not widely available and expensive. In recent studies it has been demonstrated that MRI images obtained are clear, and cross sections in several anatomic planes can be obtained without the need for contrast material [6]. As described in the literature, also the last type of CT scans provide more information than echocardiography: detailed information about the calcification, water matter, heterogenousity of content, ability to measure the densities etc. [7], but MRI is the most reliable diagnostic procedure [6].

The results of the present report suggest, that contrast echocardiography and RT3DE can help in the correct diagnosis of hydatid disease, in 
equivocal multicystic cases. Further investigations are needed to clarify its value, mainly in difficult cases.

\section{References}

1. Thameur H, Abdelmoula S, Chenik S, Bey M, Ziadi M, Mestiri T, et al. (2001) Cardiopericardial hydatid cysts. World J Surg 25(1):58-67

2. Makaryus AN, Hametz C, Mieres J, Kort S, Carneglia J, Mangion J (2004) Diagnosis of suspected cardiac echinococcosis with negative serologies: role of transthoracic, transesophageal, and contrast echocardiography. Eur J Echocardiogr 5(3):223-227

3. Barbetseas J, Lambrou S, Aggeli C, Vyssoulis G, Frogoudaki A, Tsiamis E et al (2005) Cardiac hydatid cysts: echocardiographic findings. J Clin Ultrasound 33(4):201-205

4. Atilgan D, Kudat H, Tukek T, Ozcan M, Yildirim OB, Elmaci TT et al (2002) Role of transesophageal echocardiography in diagnosis and management of cardiac hydatid cyst: report of three cases and review of the literature. J Am Soc Echocardiogr 15(3): 271-274

5. Sinha A, Nanda NC, Panwar RB, Kasliwal RR, Chauhan N, Beniwal S et al (2004) Live three-dimensional transthoracic echocardiographic assessment of left ventricular hydatid cyst. Echocardiography 21(8):699-705

6. Desnos M, Brochet E, Cristofini P, Cosnard G, Keddari M, Mostefai M et al (1987) Polyvisceral echinococcosis with cardiac involvement imaged by two-dimensional echocardiography, computed tomography and nuclear magnetic resonance imaging. Am J Cardiol 59(4):383-384

7. Gross BH, Glazer GM, Francis IR. (1983) CT of intracardiac and intrapericardial masses. AJR Am J Roentgenol 140(5):903-907

8. Vuitton DA, Pawlowski PZ (2001) Echinococcosis in humans: clinical apects, diagnosis and treatment. In: WHO/OIE Manual on Echinococcosis in Humans and Animals: a public health problem of global concern. Office International des Epizooties, Paris

9. Ghannad E, Abbou CB, Nottin R, Hourdebaigt-Larrusse P, Soulie J, Grivaux M (1983) [Cardiac hydatidosis]. Sem Hop 59(19):1459-1463

10. Lidove O, Chauveheid MP, Papo T, Vuitton DA, Piarroux R, Hernigou A et al (2005) Echinococcus multilocularis massive pericardial infection: late and dramatic improvement under albendazole therapy. Am J Med 118(2):195-197

11. Gottstein B, Jacquier P, Bresson-Hadni S, Eckert J (1993) Improved primary immunodiagnosis of alveolar echinococcosis in humans by an enzyme-linked immunosorbent assay using the Em2plus antigen. J Clin Microbiol 31(2):373-376 